\title{
Biomarkers of inflammation and repair in kidney disease progression
}

\author{
Jeremy Puthumana, ${ }^{1}$ Heather Thiessen-Philbrook, ${ }^{2}$ Leyuan Xu, ${ }^{1}$ Steven G. Coca, ${ }^{3}$ Amit X. Garg, ${ }^{4}$ Jonathan Himmelfarb, ${ }^{5}$ \\ Pavan K. Bhatraju, ${ }^{6}$ T. Alp Ikizler, ${ }^{7}$ Edward D. Siew, ${ }^{7,8}$ Lorraine B. Ware, ${ }^{9}$ Kathleen D. Liu, ${ }^{10}$ Alan S. Go, ${ }^{10,11}$ James S. Kaufman, ${ }^{12}$ \\ Paul L. Kimmel, ${ }^{13}$ Vernon M. Chinchilli, ${ }^{14}$ Lloyd G. Cantley, ${ }^{1}$ and Chirag R. Parikh ${ }^{2}$
}

\begin{abstract}
'Department of Internal Medicine, Yale School of Medicine, New Haven, Connecticut, USA. ²Division of Nephrology, School of Medicine, Johns Hopkins University, Baltimore, Maryland, USA. ${ }^{2}$ Division of Nephrology, Icahn School of Medicine at Mount Sinai, New York, New York, USA. ${ }^{4}$ Division of Nephrology, Department of Medicine, Western University, London, Ontario, Canada. ${ }^{5}$ Kidney Research Institute and ${ }^{6}$ Division of Pulmonary and Critical Care Medicine, Department of Medicine, University of Washington, Seattle, Washington, USA. 'Division of Nephrology \& Hypertension, Department of Medicine, Vanderbilt University Medical Center, Nashville, Tennessee, USA. ${ }^{8}$ Tennessee Valley Health Services, Nashville Veterans Affairs Hospital, Nashville, Tennessee, USA. ${ }^{9}$ Division of Allergy, Pulmonary and Critical Care Medicine, Department of Medicine, Vanderbilt University Medical Center, Nashville, Tennessee, USA. ${ }^{10}$ Division of Nephrology, UCSF School of Medicine, San Francisco, California, USA. "Division of Research, Kaiser Permanente Northern California, Oakland, California, USA. ${ }^{12}$ Division of Nephrology, Veterans Affairs New York Harbor Health Care System, New York University School of Medicine, New York, New York, USA. ${ }^{13}$ Division of Kidney, Urologic, and Hematologic Diseases, National Institute of Diabetes, Digestive and Kidney Diseases (NIDDK), NIH, Bethesda, Maryland, USA. ${ }^{14}$ Department of Public
\end{abstract} Health Sciences, Pennsylvania State University College of Medicine, Hershey, Pennsylvania, USA.

INTRODUCTION. Acute kidney injury and chronic kidney disease (CKD) are common in hospitalized patients. To inform clinical decision making, more accurate information regarding risk of long-term progression to kidney failure is required.

METHODS. We enrolled 1538 hospitalized patients in a multicenter, prospective cohort study. Monocyte chemoattractant protein 1 (MCP-1/CCL2), uromodulin (UMOD), and YKL-40 (CHI3L1) were measured in urine samples collected during outpatient follow-up at 3 months. We followed patients for a median of 4.3 years and assessed the relationship between biomarker levels and changes in estimated glomerular filtration rate (eGFR) over time and the development of a composite kidney outcome (CKD incidence, CKD progression, or end-stage renal disease). We paired these clinical studies with investigations in mouse models of renal atrophy and renal repair to further understand the molecular basis of these markers in kidney disease progression.

RESULTS. Higher MCP-1 and YKL-40 levels were associated with greater eGFR decline and increased incidence of the composite renal outcome, whereas higher UMOD levels were associated with smaller eGFR declines and decreased incidence of the composite kidney outcome. A multimarker score increased prognostic accuracy and reclassification compared with traditional clinical variables alone. The mouse model of renal atrophy showed greater $C \mathrm{cl} 2$ and $C h i 3 / 1$ mRNA expression in infiltrating macrophages and neutrophils, respectively, and evidence of progressive renal fibrosis compared with the repair model. The repair model showed greater Umod expression in the loop of Henle and correspondingly less fibrosis.

CONCLUSIONS. Biomarker levels at 3 months after hospitalization identify patients at risk for kidney disease progression.

FUNDING. NIH.

Conflict of interest: SGC reports personal income and equity and stock options from RenaltyixAl and pulseData; he also reports personal income from 3ive, Bayer, Boehringer-Ingelheim, CHF Solutions, inRegen, Quark, Relypsa, and Takeda. JH reports personal income from Akebia Therapeutics, Chinook Therapeutics, Maze Therapeutics, Pfizer, RenalytixAl, and Seattle Genetics. EDS reports personal income from Akebia Therapeutics, Da Vita, and UpToDate; he also serves as an associate editor for the Clinical Journal of the American Society of Nephrology. LBW reports personal incoime from Bayer, Boehringer Ingelheim, Citius, CSL Behring, Foresee, Merck, and Quark; she also received research funding from CSL Behring and Genentech. KDL reports personal income from Astra Zeneca, Baxter, Biomerieux, Durect, Potrero Med, Quark, Theravance, and UpToDate; she also holds stock in Amgen and is an associate editor at the American Thoracic Society. PLK reports being an editor of the textbook Chronic Renal Disease. LGC reports personal income from MPM Capital and Vivace Therapeutics. CRP reports personal income and equity and stock options from RenaltyixAl; he also reports personal income from Genfit Biopharmaceutical Company and Akebia Therapeutics.

Role of funding source: The study was supported by cooperative agreements from the NIDDK. Representatives from the NIDDK participated in all phases of the study and approved the final manuscript for submission.

Copyright: (c) 2021, American Society for Clinical Investigation.

Submitted: May 5, 2020; Accepted: December 1, 2020; Published: February 1, 2021. Reference information: / Clin Invest. 2021;131(3):e139927. https://doi.org/10.1172/JCI139927.

\section{Introduction}

Acute kidney injury (AKI) is a complex disorder associated with increased risk of morbidity and mortality. AKI has long been believed to be a fully reversible condition. However, recent studies have shown that patients who survive an episode of AKI may experience persistent subclinical kidney injury, placing them at higher risk for progression to chronic kidney disease (CKD), end-stage-renal disease (ESRD), and death (1-3). The principal response to kidney injury is the development of inflammation, which can lead to renal fibrosis. Depending on the extent and duration of injury, inflammation and fibrosis can either lead to ESRD or stabilize with little loss of kidney function (4). Biomarkers associated with renal inflammation and repair may be helpful in distinguishing patients at risk for kidney failure from those who are likely to recover function.

YKL-40, also known as chitinase 3-like 1 (CHI3L1) and encoded by the Chi3l1 gene, and monocyte chemoattractant protein 1 (MCP-1), also referred to as chemokine C-C ligand 2 (CCL2) and 
Table 1. Characteristics of participants by AKI status at index hospitalization

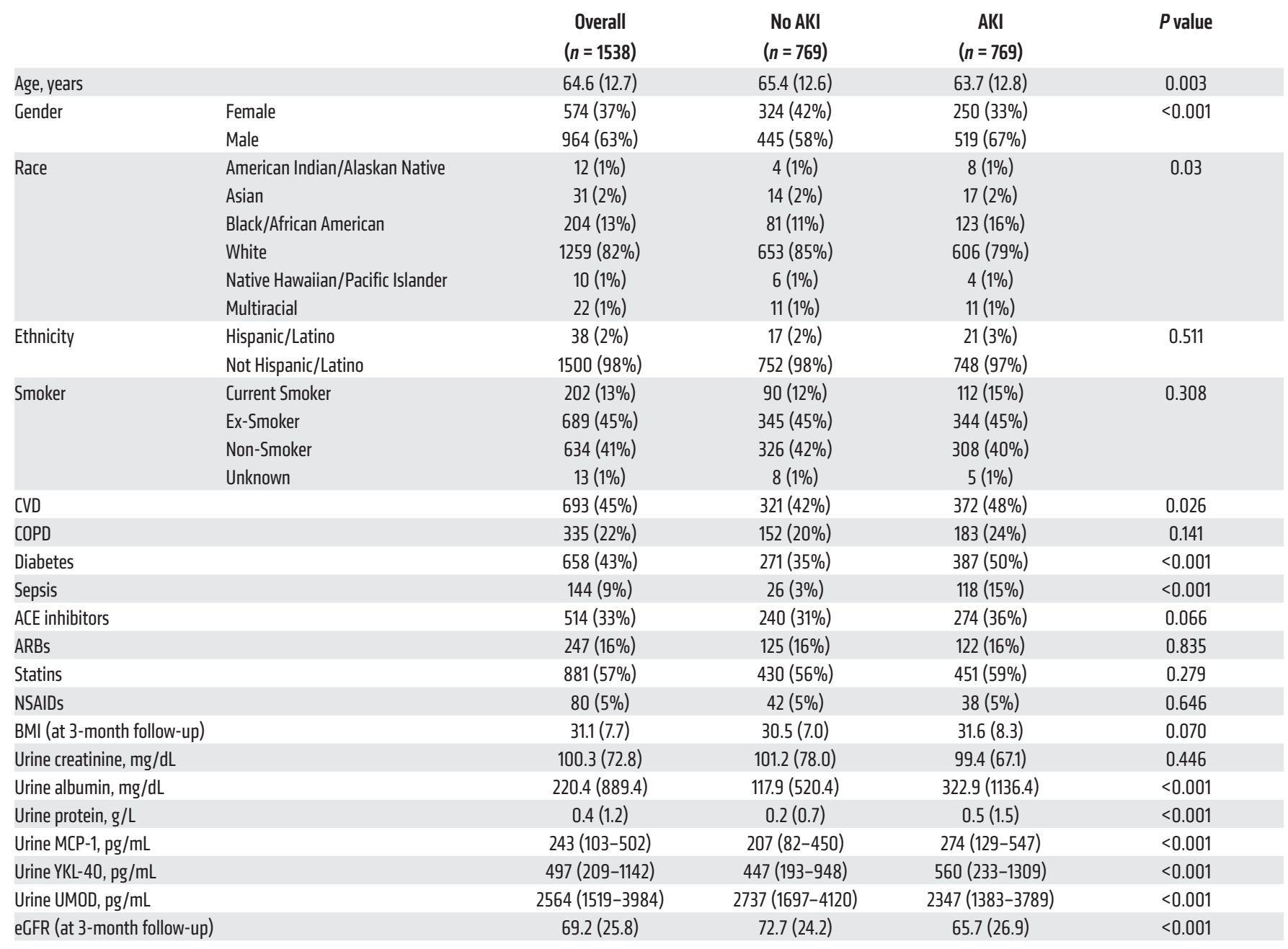

Values reported are mean (SD), median (IQR), or $n$ (\%). AKI, acute kidney injury; CVD, cardiovascular disease; COPD, chronic obstructive pulmonary disease; ACE, angiotensin-converting enzyme; ARBs, angiotensin II receptor blockers; NSAIDs, nonsteroidal antiinflammatory drugs; BMI, body mass index; eGFR, estimated glomerular filtration rate.

encoded by the $\mathrm{Ccl} 2$ gene, are emerging biomarkers in kidney disease. Urine YKL-40 and MCP-1 have been shown to be indicators of structural kidney damage and inflammation in experimental and clinical settings (5-11). While these proteins are produced in response to ischemic and toxic kidney injury, uromodulin (UMOD), encoded by the Umod gene, is a mucoprotein primarily synthesized by the thick ascending limb of the loop of Henle (TAL) associated with improved tubular function in the general population and lower risk of AKI in patients after cardiac surgery $(12,13)$.

Although many studies have investigated biomarkers in the early detection of AKI and for predicting short-term outcomes, few studies have examined their ability to predict long-term kidney function. Furthermore, previous studies have largely relied on biomarker measurements taken in hospitalized patients during an episode of AKI. However, individual biomarkers have been observed to have intricate and dynamic levels in this setting due to acute changes in cellular metabolism, vascular function, and inflammation. Consequently, due to their dynamic nature, biomarker levels during hospitalization may not accurately indicate the risk for long-term disease progression and may be challenging to incorporate into clinical practice. Therefore, we conducted a prospective, multicenter cohort study to assess whether elevations of urinary biomarkers of inflammation and repair at 3 months after hospitalization could identify which patients would have long-term glomerular filtration rate (GFR) decline, CKD progression, ESRD, and death after hospitalization with or without clinical AKI. We paired these clinical studies with investigations of 2 separate murine models of renal atrophy and renal repair, respectively, to better understand the molecular basis of these biomarkers in the progression of CKD.

\section{Results}

Baseline characteristics of the population stratified by clinical AKI status at the time of index hospitalization are presented in Table 1. By design, among the 1538 participants in the study, 769 (50\%) had clinical AKI (as defined by rise in serum creatinine) at the 

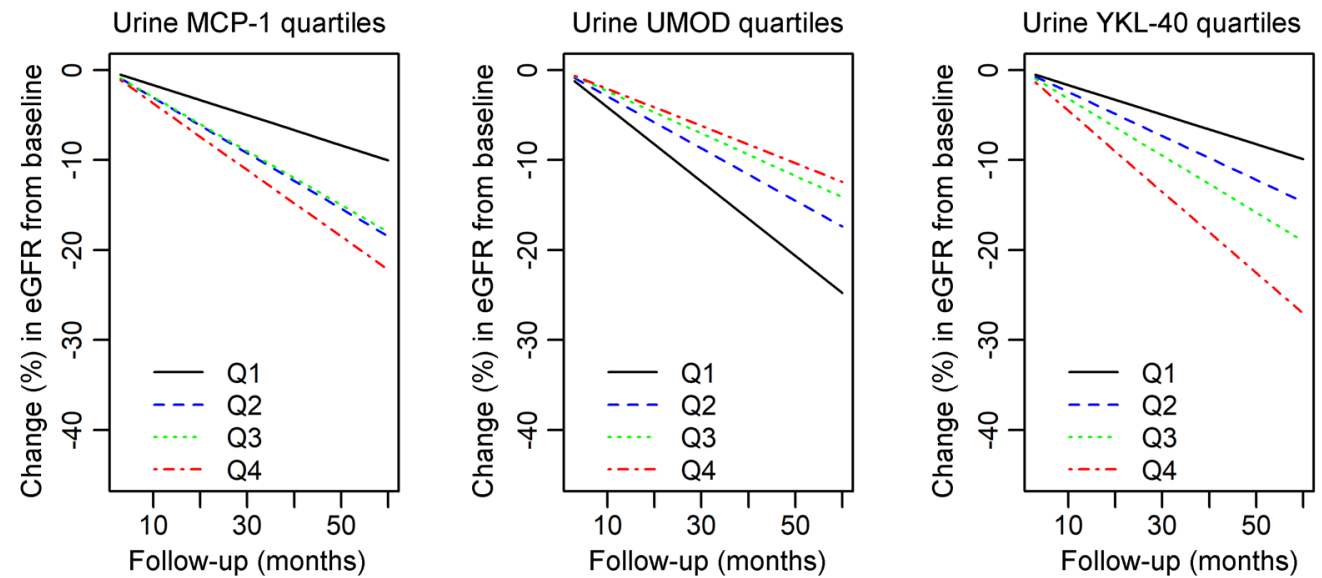

Figure 1. Levels of biomarkers and change in eGFR from baseline study visit 3 months after discharge. Adjusted for AKI and CKD status at index hospitalization, gender, black race, Hispanic ethnicity, smoking status, diabetes, sepsis during index hospitalization, body mass index at 3-month in-person visit, $\log _{2}$-transformed urine creatinine and albumin at the 3-month in-person visit, and eGFR determined at 3-month in-person visit.

time of index hospitalization and 769 (50\%) did not. In the overall cohort, the median MCP-1 level was $243 \mathrm{pg} / \mathrm{mL}$ (IQR, 103-502), YKL-40 was $497 \mathrm{pg} / \mathrm{mL}$ (IQR, 209-1142), and UMOD was 2564 $\mathrm{pg} / \mathrm{mL}$ (IQR, 1519-3984) at the in-person baseline study visit 3 months after discharge (Table 1).

Correlation coefficients and effect modification. The correlation between urinary YKL-40 and MCP-1 was moderate in strength $(r$ $=0.62 ; P<0.001)$, whereas urinary UMOD had little to no correlation $(r<0.05)$ with the other biomarkers (Supplemental Table 1 ; supplemental material available online with this article; https:// doi.org/10.1172/JCI139927DS1). There was no statistically significant effect modification by clinical AKI status on the association between urine biomarkers and the composite kidney outcome or mortality. In other words, urine biomarkers were associated with kidney disease progression and mortality similarly in those with and without a history of clinical AKI during hospitalization (Supplemental Table 2).

Relationship between biomarker levels and change in eGFR during follow-up. We determined the relationship between the urine biomarker levels at baseline and long-term change in estimated GFR (eGFR). The decline in eGFR was greater in participants with higher baseline levels of urine MCP-1 and YKL-40, whereas there was a lesser decline in eGFR among participants with higher baseline levels of urine UMOD (Figure 1). Participants in the highest MCP-1 and YKL-40 quartile had significantly greater decline in eGFR than those in the lowest biomarker quartile in adjusted analyses. Over a period of 4 years, the decline in eGFR was 8.0\% (95\% CI, 7.1-9.0) in the lowest quartile of MCP-1, as compared with $17.8 \%$ (95\% CI, 16.7-18.8) in the highest quartile. The decline in eGFR was $7.9 \%$ (95\% CI, 7.0-8.9) in the lowest quartile versus $21.7 \%$ (95\% CI, 20.622.7) in the highest quartile for YKL-40. In contrast, over a period of 4 years, the decline in eGFR was $19.8 \%$ (95\% CI, 18.8-20.8) in and mortality

the lowest quartile of UMOD, as compared with 9.9\% (95\% CI, 9.0-10.9) in the highest quartile (Supplemental Table 3).

Composite kidney outcome. Of the 1538 patients in the study, $300(20 \%)$ reached the composite kidney outcome (CKD incidence, CKD progression, or development of ESRD) during follow-up. We examined whether urinary biomarkers at baseline were associated with the composite kidney outcome. In adjusted analyses, MCP-1 (HR, 1.32 for each doubling; 95\% CI, 1.18-1.46) and YKL-40 (HR, 1.15 for each doubling; 95\% CI, 1.09-1.22) were positively associated with the composite kidney outcome. In contrast, UMOD was associated with decreased rates of the composite kidney outcome (HR, 0.85 for each doubling; 95\% CI, 0.74-0.97) (Table 2). The association of urine biomarkers with the time to reach the composite kidney outcome are displayed using Kaplan-Meier curves in Figure 2. Because the interaction of clinical AKI status with the associations between urine MCP1 and YKL-40 and the composite kidney outcome showed trends toward significance, we also provided stratified analyses for these biomarkers by AKI status. In adjusted analyses, MCP-1 and YKL40 were positively associated with the composite kidney outcome in patients with and without AKI (Supplemental Table 4).

Table 2. Associations between biomarkers and the composite kidney outcome

Biomarker
( $\log _{2}$ transformed)

MCP-1

UMOD

YKL-40

\begin{tabular}{|c|c|}
\hline $\begin{array}{r}\text { Composite } \\
\text { HR }\end{array}$ & $\begin{array}{l}\text { ley Outcome } \\
\text { CI) }\end{array}$ \\
\hline Unadjusted & Adjusted $^{\mathrm{B}}$ \\
\hline $1.23(1.14-1.33)$ & $1.32(1.18-1.46)$ \\
\hline $0.68(0.60-0.77)$ & $0.85(0.74-0.97)$ \\
\hline $1.27(1.20-1.34)$ & 1.15 (1.09-1.22) \\
\hline
\end{tabular}
increase in $\log _{2}$-transformed urine biomarker concentrations. ${ }^{B}$ Adjusted for AKI and CKD status at index hospitalization, gender, black race, Hispanic ethnicity, smoking status, diabetes, sepsis during index hospitalization, body mass index at 3 -month in-person visit, $\log _{2}$-transformed urine creatinine and albumin at the 3-month in-person visit, eGFR determined at 3-month in-person visit. 

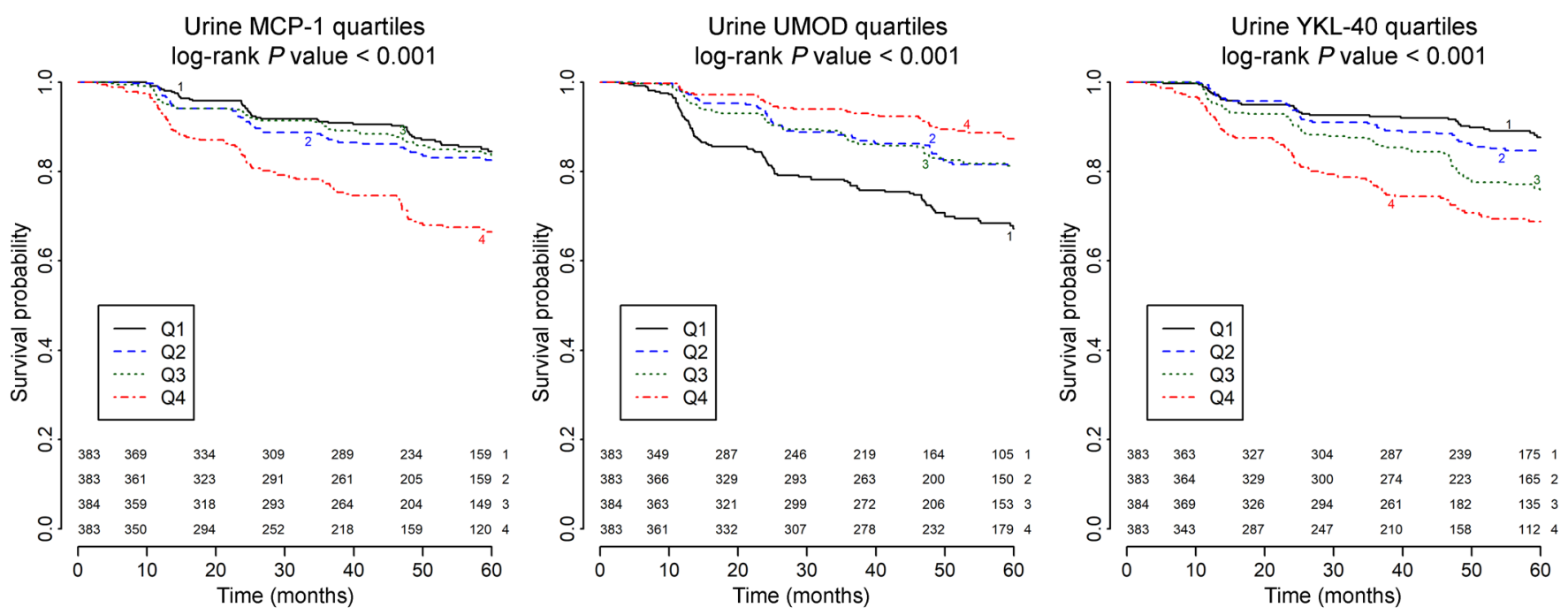

Figure 2. Kaplan-Meier curves of the proportion of patients reaching the composite kidney outcome relative to time from baseline by biomarker quartiles.

Mortality. During follow-up, 320 (21\%) participants died. Each urine biomarker was assessed as a predictor of mortality. The association between biomarkers and mortality is shown in Figure 3. In adjusted analyses, MCP-1 (HR, 1.32 for each doubling; $95 \%$ CI, 1.20-1.45) and YKL-40 (HR, 1.09 for each doubling; 95\% CI, 1.03-1.15) were associated with mortality. However, there was no significant association between urine UMOD and mortality (HR, 1.07 for each doubling; 95\% CI, 0.94-1.22) (Table 2).

Biomarker score. A biomarker score was constructed by assigning integer values to each biomarker quartile. The biomarker score had a range of 0-9 and was calculated for each patient by summing the integer values assigned for the 3 biomarker levels for that patient (Supplemental Table 5).

The mean event rate per 1000 patient-years increased from 21.3 (95\% CI, 10.6-42.5) in patients with a biomarker score of 0 to 100.8 (95\% CI, 10.2-1000.0) in patients with a biomarker score of 8-9. Patients with the highest score had an over 4 -fold increase in event rate, compared with the lowest score, after adjusting for clinical variables (Supplemental Table 6). The association between biomarker score and the composite kidney outcome is displayed using a Kaplan-Meier curve in Supplemental Figure 1. Adding the biomarker score to the clinical model incrementally increased the AUC to 0.835 (95\% CI, 0.799-0.871) compared with the clinical model alone $(P<0.01)$. Combining the biomarker score and clinical model also significantly enhanced reclassification compared with the clinical model alone; the net reclassification improvement (NRI) was 0.078 (95\% CI, 0.002-0.122) (Table 3).

We evaluated the effect that screening patients for an elevated biomarker score would have on the total sample size of a clinical trial for an intervention intended to prevent CKD. Our hypothetical clinical trial included a 12-month accrual period and 36-month follow-up; it specified $90 \%$ power to detect an HR of 0.8 using 2-sided testing and $\alpha$ equal to 0.05. Supplemental Table 7 illustrates how the total sample size would be reduced if only patients
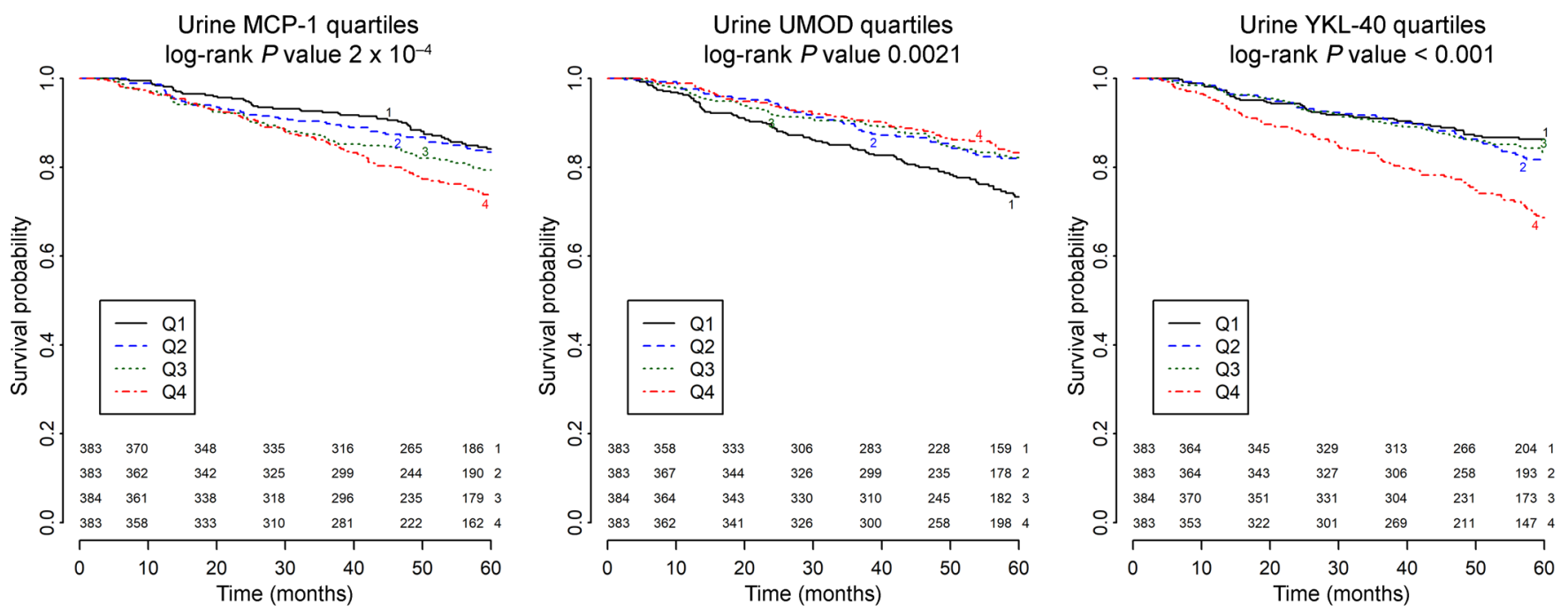

Figure 3. Kaplan-Meier curves of the proportion of patients surviving relative to time from baseline by biomarker quartiles. 


\section{Table 3. Discrimination and reclassification for composite kidney outcome}

$\begin{array}{lcc} & \text { AUC (95\% Cl) } & \text { NRI (95\% Cl) } \\ \text { Clinical Model }^{A} & 0.815(0.762-0.850) & \\ \text { Clinical Model }^{A}+\text { Biomarker Score } & 0.835(0.799-0.871) & 0.078(0.002-0.122) \\ & P<0.01 & \end{array}$

AUC, area under the curve; NRI, net reclassification improvement. AIncluded AKI and CKD status at index hospitalization, gender, black race, Hispanic ethnicity, smoking status, diabetes, sepsis during index hospitalization, body mass index at 3-month in-person visit, $\log _{2}-$ transformed urine creatinine and albumin at the 3-month in-person visit, eGFR determined at 3-month in-person visit.

above a specific biomarker score were enrolled. If only patients with a biomarker score of 8-9 were enrolled, it would allow investigators to observe a treatment effect in only 2605 patients, while a study without prognostic enrichment would require 8027 patients.

Ccl2, Chi3l1, and Umod mRNA levels correlate with atrophy and renal fibrosis in a mouse model of kidney injury. To understand the renal parenchymal implications of changes in urinary MCP-1, YKL40 , and UMOD, we assessed mRNA expression of the corresponding genes (Ccl2, Chi3l1, and Umod, respectively) in 2 mouse models in which ischemia/reperfusion injury (IRI) is followed by repair or atrophy, as previously described (14-16). Consistent with previous studies, the atrophy model resulted in a significant increase in PDGFR $\beta$-positive myofibroblast accumulation and renal interstitial fibrosis and a decrease in outer medullary thickness 30 days after surgery compared with the repair model (Figure 4, A-E) (14-16). As shown in Figure 4F, there was increased gene expression of major fibrotic components including collagen $\alpha-1(\mathrm{I})$, fibronectin, and PDGFR $\beta$ (Pdgfrb, myofibroblast marker) in the atrophy model compared with the repair model. Consistent with the MCP-1, YKL-40, and UMOD levels in human urine, quantitative PCR of whole-kidney mRNA revealed that both Ccl2 and Chi3l1 were significantly higher in the setting of progressive kidney fibrosis and atrophy, whereas Umod levels were higher in the setting of kidney repair (Figure $4 \mathrm{G}$ and Supplemental Table 8). Single-cell RNA sequencing (scRNA-seq) analysis of these 2 models 14 days after IRI showed that Ccl2, Chi3l1, and Umod were expressed by the infiltrating macrophages, neutrophils, and TAL/distal convoluted tubule (DCT) cells, respectively, and that expression of Ccl2 and Chi3l1 was greater in the atrophy model, whereas expression of Umod was greater in the repair model (Figure 5). Pearson analysis of Ccl2, Chi3l1, and Umod and fibrosis-related genes showed a strong correlation between $\mathrm{Ccl} 2$ and all 3 fibrosis-related genes analyzed and a moderate correlation between Chi3l1 and all 3 fibrosis-related genes analyzed (Supplemental Table 9).

\section{Discussion}

In this prospective cohort study, we evaluated the association of 3 kidney biomarkers measured at 3 months after hospitalization with the change in eGFR over time, a composite kidney outcome (CKD incidence, CKD progression, or ESRD), and death. We identified strong associations between higher urinary MCP-1 and YKL40 and eGFR decline over time, the composite kidney outcome, and all-cause mortality. In contrast, we identified a significant association between higher urinary UMOD and a smaller eGFR decline over time and fewer renal outcomes. To understand the renal parenchymal implications of changes in these biomarkers, we performed molecular and histological characterization of 2 separate murine models of IRI followed by repair and atrophy, respectively. scRNA-seq analysis of these 2 complementary mouse models showed that Ccl2, Chi3l1, and Umod were expressed by the infiltrating macrophages, neutrophils, and TAL/DCT cells, respectively. In our mouse model of renal atrophy, there was greater expression of $\mathrm{Ccl} 2$ and Chi3l1 mRNA and evidence of myofibroblast accumulation and progressive renal fibrosis compared with the repair model, whereas the repair model showed greater expression of Umod and correspondingly less myofibroblast accumulation and fibrosis.

The primary response to kidney injury is the simultaneous initiation of injury and repair mechanisms. Basile and colleagues divide the clinical course of kidney injury into 3 phases: the development phase, extension phase, and resolution phase (17). The development phase represents the immediate effects of the insult. Depending on the extent and duration of injury and underlying renal reserve, the initial insult may manifest with little or no change in kidney function (as measured through serum creatinine) and may only be detected through sensitive kidney-specific biomarkers. At this point, mild injury may resolve, or if the injury is severe enough, may extend and result in increased renal impairment. The resolution phase represents the final net result of injury and repair mechanisms. If repair mechanisms keep pace with injury, there may be functional resolution over time; however, if ongoing injury surpasses the kidney's repair mechanisms, the end result is fibrosis and durable reduction in renal function.

Previous literature shows that persistent renal inflammation is the main driver of the extension phase $(18,19)$. In particular, the inflammatory process is characterized by recruitment and activation of leukocytes including macrophages, neutrophils, and T cells, which in turn can result in the production of profibrotic cytokines and growth factors. Together, these profibrotic factors can promote myofibroblast activation and pathogenic collagen deposition (20). Our mouse models of renal atrophy and repair build on these findings, showing that $\mathrm{Ccl} 2$ and Chi3l1 were expressed predominantly by infiltrating macrophages and neutrophils, respectively, and were associated with myofibroblast accumulation and interstitial kidney fibrosis, the histopathological hallmarks of CKD.

In our large, multicenter cohort study, these biomarkers were present in the urine of many patients 3 months after hospitalization. This finding may indicate ongoing inflammation, tubular injury, and fibrosis extending beyond hospitalization. The association between elevations in these biomarkers and progressive renal decline in adjusted analyses suggests these maladaptive repair processes may play an important role in the development of CKD. In addition, the association between increased biomarkers and renal decline was observed across all hospitalized patients regardless of AKI status at enrollment, suggesting that renal decline was not limited to those with clinical AKI (as measured through serum creatinine), but that even those with subclinical kidney injury (an increase in biomarkers of tissue injury without a simultaneous increase in creatinine) may progress to CKD. Elevated injury biomarkers after hospital- 
A

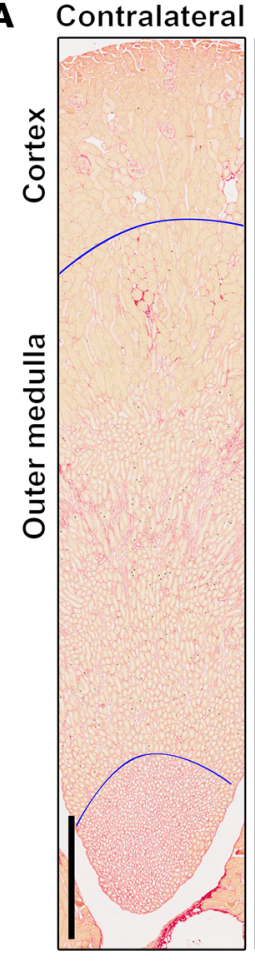

C

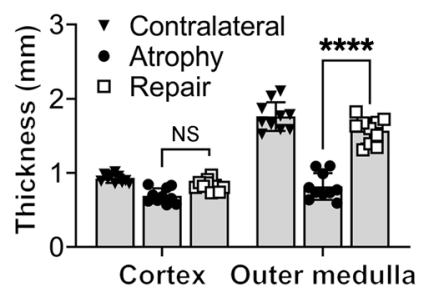

$\mathbf{F}$

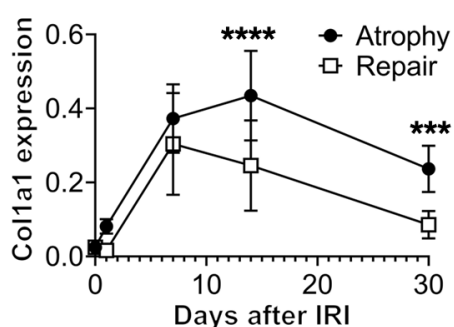

G
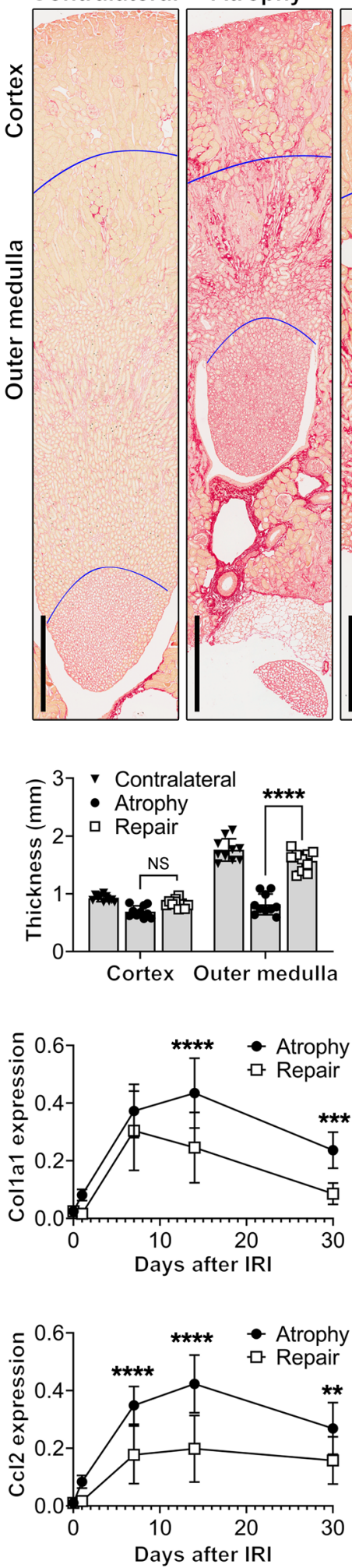

Repair

D
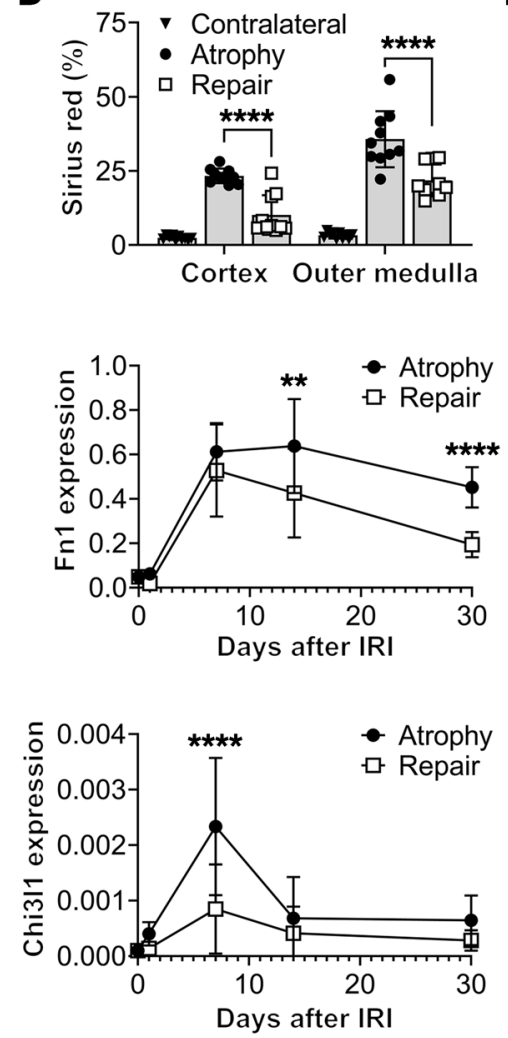

B

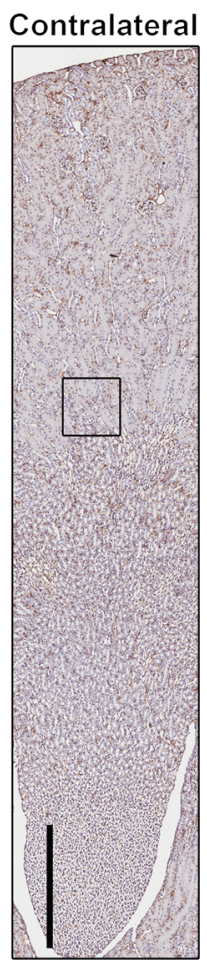

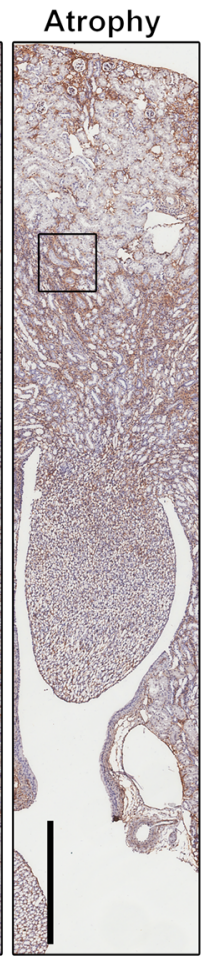
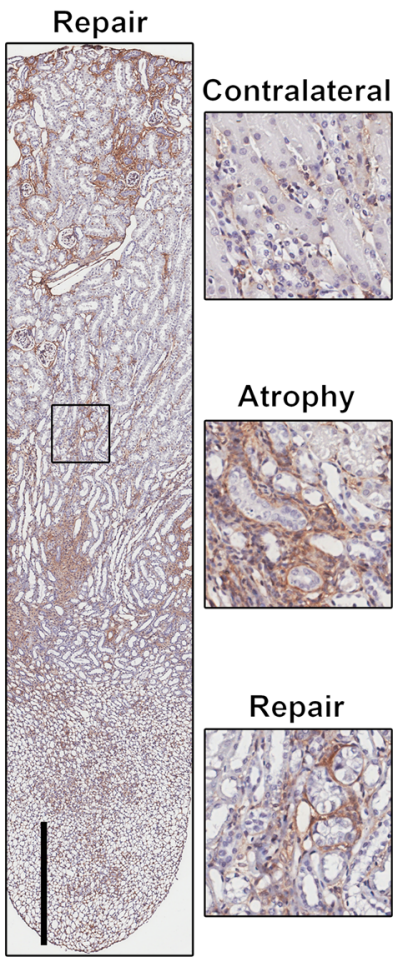

E
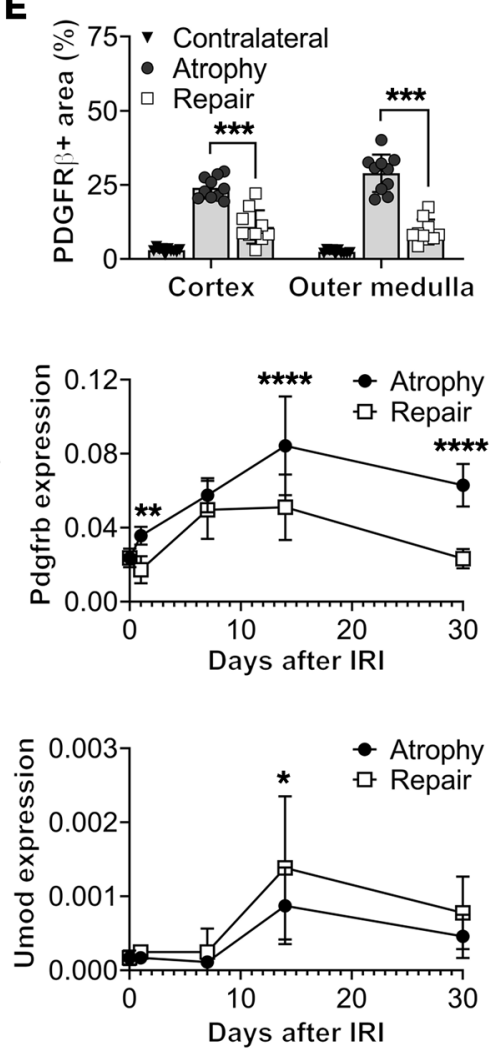

Figure 4. Kidney fibrosis and atrophy are preceded by upregulation of $\mathbf{C} \boldsymbol{c l} \mathbf{2}$ and $\mathbf{C h i 3 / 1}$. Wild-type mice were subjected to 27 minutes of unilateral IRI with contralateral kidney intact (atrophy model) or unilateral IRI with contralateral nephrectomy (repair model). The mice were sacrificed on days 1, 7, 14, and 30 after injury. Contralateral, atrophy, and repair kidneys were harvested at 30 days after injury, and kidney sections were stained with Picrosirius red to detect collagen deposition and immunostained with anti-PDGFR $\beta$ antibody to detect interstitial myofibroblasts. (A and B) Representative images of kidney sections. Scale bars: $500 \mu \mathrm{m}$. (C-E) Quantification of cortical and medullary thickness, Picrosirius red area, and PDCFR $\beta$-positive area, respectively, $n=10$ kidneys/model. $P<0.0001$ among group means and ${ }^{* * *} P<0.001,{ }^{* * *} P<0.0001$ by 2 -way ANOVA in the indicated subgroup analyses. (F and $\mathbf{G}$ )

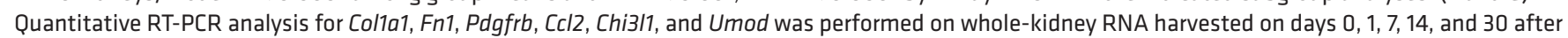

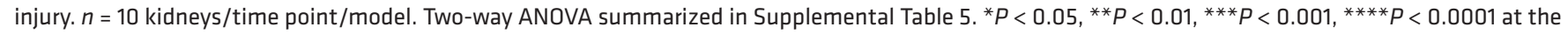
indicated time point. NS, not significant. 


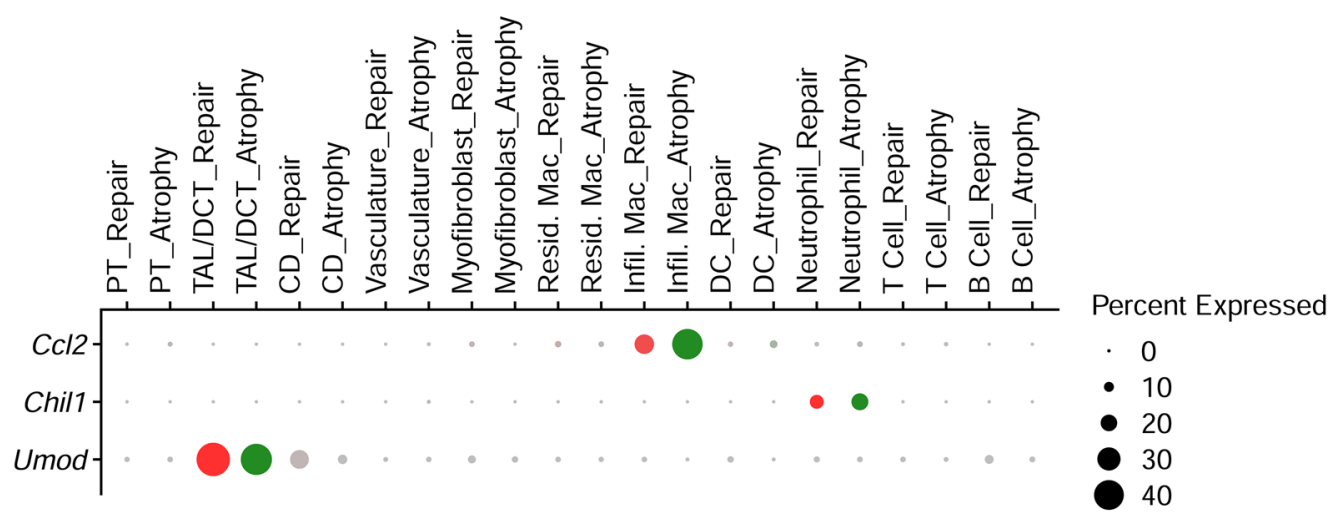

Figure 5. Single-cell RNA sequencing analysis of kidneys from atrophy and repair models for biomarker gene expression. Atrophy and repair kidneys were harvested at 14 days after injury for cell isolation followed by single-cell RNA sequencing analysis. Cell clustering and data analysis were performed using Seurat v3.1.5 R package. Relevant biomarker gene expression is shown as a dot plot. PT, proximal tubule; TAL, thick ascending limb of the loop of Henle; DCT, distal convoluted tubule; CD, collecting duct; DC, dendritic cells.

ization could trigger further diagnostic work-up and allow for targeted lifestyle interventions and medical optimization.

UMOD, a mucoprotein synthesized by the TAL, has been shown to be a marker of renal reserve and play a key role in the innate immunity of the kidney $(13,21)$. Animal studies have shown that UMOD-knockout mice have an increased susceptibility to urinary tract infection (22). In addition, recent genome-wide association studies have linked polymorphisms in UMOD with an increased risk of CKD and diabetic nephropathy $(23,24)$. In our study, higher levels of UMOD were independently associated with a smaller decline in eGFR and reduced risk of long-term renal outcomes. Consistent with these clinical findings, Umod expression was significantly higher in cells of the TAL/DCT in our mouse model of kidney repair. Our findings, taken together with the literature, corroborate UMOD's role as an important protective factor in maintaining kidney function and that UMOD may be indicative of adaptive tubular repair. Decreased levels of UMOD after hospitalization could help identify patients at risk for decreased renal function.

When added to the clinical model, our biomarker score increased prognostic accuracy by improving the AUC and NRI. Our results reinforce the concept that multiple biomarkers may be useful to assess the risk that results from the complex interplay between renal repair and atrophy. In future studies, we hope to identify and measure biomarkers in additional independent disease pathways to further refine our biomarker score.

Drug development is a lengthy and expensive process. For many diseases, including CKD, efficiently testing drugs has been a major challenge. Accordingly, the US Food and Drug Administration (FDA) has recently released final guidance documents on enrichment strategies for clinical trials (25). By using prognostic biomarkers to select patients at high risk for a disease-related endpoint, it is possible to considerably reduce the sample size needed to adequately power a study to detect a drug's effectiveness. These strategies have successfully been employed in cardiovascular and oncology trials (25). However, no prognostic biomarkers have been used as enrollment criteria in clinical trials of CKD. In a hypothetical clinical trial for an intervention intended to prevent CKD, we show that, by enrolling only patients above a specific biomarker level, it may be possible to observe significant treatment effects in much smaller clinical trials.
Major strengths of our study include its large, multicenter, prospective design as well as systematic, long-term follow-up for relevant outcomes. Previous studies have been limited by small sample sizes and retrospective designs, which have hampered their ability to determine the independent association between biomarkers and long-term outcomes. Furthermore, previous studies have relied on biomarker measurements taken during index hospitalization. However, individual biomarker levels have been observed to vary considerably in this setting, limiting their ability to accurately predict long-term disease progression. Our study measures biomarkers after the acute phase, during outpatient follow-up, allowing them to predict long-term outcomes. Moreover, this is a highly clinically relevant time point, as physicians may follow patients in the outpatient setting at this time after a recent hospitalization. Therefore, biomarker measurements during outpatient follow-up can serve as a useful screening tool for physicians.

Our study also has several limitations worth considering. Our study did not differentiate the various etiologies of AKI. In addition, we only included centers in North America, and therefore our results may not be generalizable to all hospitalized patients. Although we adjusted for multiple patient characteristics, we cannot rule out residual confounding. Finally, the study did not collect biopsy samples from patients due to the invasiveness of the procedure and potential risks for complications. Therefore, we were not able to assess the association of these inflammatory biomarkers in human biopsy specimens with long-term outcomes. However, prior studies from kidney transplant patients (a related population that often receives surveillance biopsies) demonstrates that numbers of inflammatory cells present on posttransplant biopsies correlated with progression of fibrosis and long-term eGFR decline (26-28). Future studies will be needed to assess these markers in biopsy tissue in patients after hospitalization.

In conclusion, CKD currently affects $15 \%$ of the US population and is expected to become more prevalent in decades to come, underscoring the need to implement effective screening and prevention strategies (29). Monitoring biomarker levels after hospitalization could help identify subgroups of patients at risk for progressive decline in kidney function. Further studies will be needed to assess whether closer follow-up and targeted interventions can improve outcomes in this population. 


\section{Methods}

Study population. The Assessment, Serial Evaluation, and Subsequent Sequelae of Acute Kidney Injury (ASSESS-AKI) Study is a prospective, matched cohort study of hospitalized patients who did or did not experience an episode of AKI and survived to complete an in-person baseline study visit 3 months after discharge. This first outpatient visit is referred to as "baseline" throughout the manuscript. Study details have been described previously (30). Briefly, hospitalized adults were enrolled between December 2009 and February 2015 at 4 clinical centers in North America. The cohort comprised 769 hospitalized patients with AKI and 769 matched patients without AKI at the index hospitalization (Supplemental Figure 2). AKI was defined as an increase of $50 \%$ or greater or $0.3 \mathrm{mg} / \mathrm{dL}$ or greater in serum creatinine above the most recent outpatient, non-emergency department serum creatinine obtained within 7 to 365 days prior to index hospitalization.

Collection of study data. The 1538 ASSESS-AKI study participants had an in-person baseline study visit 3 months after discharge, during which clinical data were systematically collected (31). In addition, we collected blood and urine samples at the visit. Participants returned for in-patient follow-up visits 12 months after discharge and annually thereafter, and were contacted by telephone at 6-month intervals between clinic visits. Medical history, medication use, and study events were updated at each in-person visit or phone contact; blood and urine samples were collected at each in-person visit and eGFR was quantified using the CKD-EPI equation (32). Vital status was determined at each study contact through medical record review and contact with the participant's healthcare proxy.

The primary kidney outcome was a composite of CKD incidence, CKD progression, and development of ESRD. In participants without preexisting CKD at index hospitalization (eGFR $\geq 60 \mathrm{~mL}$ / $\min / 1.73 \mathrm{~m}^{2}$ ), CKD incidence was defined as the combination of $25 \%$ or greater reduction in eGFR (compared with the most recent preadmission measurement) and achieving CKD stage 3 or worse. In participants with preexisting $\mathrm{CKD}$ at the index hospitalization (eGFR $<60 \mathrm{~mL} / \mathrm{min} / 1.73 \mathrm{~m}^{2}$ ), CKD progression was defined as experiencing $50 \%$ or greater reduction in eGFR (compared with the most recent preadmission measurement) or progressing to stage 5 CKD. ESRD was defined as dialysis treatment at least once a week for at least 12 consecutive weeks, receipt of kidney transplant, or death while receiving dialysis.

Biomarker measurement. Samples were aliquoted and stored at $-80^{\circ} \mathrm{C}$ until biomarker measurements. We measured serum and urine samples for creatinine concentration using the Roche enzymatic method (Roche Diagnostics). We measured urine samples for MCP-1 and YKL-40 using a multiplex assay, and urine samples for UMOD were measured on a separate assay (Meso Scale Diagnostics), the methods of which have been described previously (33). All investigators measuring biomarkers were blinded to clinical outcomes.

Animal surgery and experimental protocol. C57BL/6 (Envigo) wildtype mice (age 9-11 weeks) were used in this work. To establish the unilateral IRI (atrophy) model, warm renal ischemia was induced using a nontraumatic microaneurysm clip (FST Micro Clamps) on the left renal pedicle for 27 minutes, leaving the right kidney intact. To establish the unilateral ischemia/reperfusion with contralateral nephrectomy (repair) model, the right kidney was surgically removed at the time of left kidney ischemia, as we have previously described $(14,15)$. Blood was obtained 1 day after the surgery. Serum creatinine was measured at the Yale George M. O'Brien Kidney Center. The mice were sacrificed on days $1,7,14$, and 30 after surgery $(n=10$ per time point for each model). Control mice were sacrificed and are represented as day $0(n=10)$.

Histology and IHC. Kidneys were fixed in 10\% neutral buffered formalin and embedded in paraffin. For detection of collagen, deparaffinized kidney sections $(5 \mu \mathrm{m})$ were rehydrated and stained with Picrosirius red in $1.3 \%$ picric acid for 1 hour. PDGFR $\beta$-positive cells were detected by IHC using primary monoclonal antibodies against PDGFR $\beta$ (3169, Cell Signaling Technology) as described previously (14). Six independent fields in the cortex and 4 independent fields in the outer medulla were analyzed per kidney. The percentage area of Picrosirius red staining and PDGFR $\beta$-positive myofibroblasts, as a measure of fibrosis, and the thickness of the cortex and outer medulla were quantified using ImageJ software (NIH).

Quantitative PCR analysis. Whole-kidney RNA was extracted with an RNeasy Mini kit (Qiagen) and reverse transcribed using the iScript cDNA Synthesis Kit (Bio-Rad Laboratories). Gene expression was determined by quantitative real-time PCR using an iCycler iQ (Bio-Rad Laboratories) and normalized to hypoxanthine-guanine phosphoribosyltransferase ( $\mathrm{Hprt}$ ). Primers used included previously published primers for Ccl2, Chi3l1, Col1a1, Fn1, Pdgfrb, and Hprt, as well as Umod forward (TGCAATCTGGCTTACTGCAC) and reverse (TGAAGCCTGAGCATTGTCTG) $(14,15)$. The data are expressed using the comparative threshold cycle $(\triangle \mathrm{CT})$ method, and the mRNA ratios are given by $2^{-\Delta C T}$.

scRNA-seq analysis. Kidneys from the atrophy and repair models were harvested at 14 days after injury for cell isolation followed by scRNA-seq. The scRNA-seq library construction and sequencing were performed at the Yale Center for Genome Analysis (YCGA). Cell Ranger version 3.0.0 was used to process Chromium single-cell 3' RNAseq output and align the Read to the mouse reference transcriptome (mm9). Cell clustering and data analysis were performed using Seurat v3.1.0 R package $(34,35)$. Red blood cells, cells with transcriptome below 200 (likely cell fragments) or greater than 3,500 (potentially cell doublets), and cells with mitochondrial gene percentage greater than $50 \%$ were excluded. Principle component analysis (PCA) was performed on the scaled data. The top 30 principal components were chosen for unsupervised cell clustering. Each cluster was screened for marker genes by differential expression analysis based on the nonparametric Wilcoxon rank sum test for all the clusters with genes expressed in at least $25 \%$ of cells either inside or outside of a cluster. Cell identity was assigned based on kidney cell- and immune cell lineage-specific marker expression: Lrp2, Slc27a2, and Kap for proximal tubule; Slc12a1, Umod, Slc12a3, and Calb1 for TAL/DCT; Atp6v1g3 and Atp6vOd2 for collecting duct; Plvap and Kdr for vasculature; Acta2, Col1a1, and Fn1 for myofibroblasts; C1qa and C1qc for resident macrophages; C1qa, C1qc, Cx3cr1, and Ccr2 for infiltrating macrophages; Itgax for dendritic cells; S1OOa 8 and S100a9 for neutrophils; $\mathrm{Cd} 3 \mathrm{e}$ and $C d 3 g$ for T cells; and $C d 79 a$ and $C d 79 b$ for B cells. The scRNA-seq data reported in this paper are available in the NCBI's Gene Expression Omnibus database (GEO GSE161758).

Statistics. Descriptive statistics are reported as mean $( \pm \mathrm{SD})$ or median (IQR) for continuous variables, and as frequency (percentage) for categorical variables. For the patient cohort, we compared differences in characteristics between those who did and those who did not experience an episode of AKI during index hospitalization. 
For continuous variables, we used either the Student's $t$ test or the Wilcoxon rank sum test, and for categorical variables, we used the $\chi^{2}$ test. Correlation between urine biomarkers was assessed with Spearman's correlation coefficients.

We used Cox regression models to estimate the associations between urine biomarkers and the primary composite kidney outcome. Biomarker concentrations were modeled as $\log _{2}$-transformed continuous variables or quartiles. The model was adjusted for the following prespecified variables: AKI and CKD status at index hospitalization, gender, black race, Hispanic ethnicity, smoking status, diabetes, sepsis during index hospitalization, and body mass index. They were further adjusted for $\log _{2}$-transformed urine creatinine and albumin at the 3-month in-person visit as well as eGFR determined at the aforementioned visit. In addition, we display the association of urine biomarkers with the composite kidney outcome and mortality using Kaplan-Meier curves. For analyses of the composite kidney outcome and mortality, we fit 2 separate models according to clinical AKI status and performed tests for effect modification between each biomarker and clinical AKI status on the composite kidney outcome and mortality, respectively.

We examined the association between biomarker levels and the change in eGFR over time using methods described previously (36). Briefly, we used linear mixed-effects modeling with a random participant-specific intercept and a random time effect, by regressing $\log$ (eGFR) against biomarker quartiles, follow-up time (months since baseline), biomarker quartiles $\times$ follow-up time, and baseline eGFR, in addition to the aforementioned covariates.

We subsequently generated a combined biomarker score, using methods described previously (37). The biomarker score was constructed by assigning integer values to each biomarker quartile. The biomarker score had a range of 0 -9 and was calculated for each patient by summing the integer values assigned for the 3 biomarker levels for that patient. The prognostic accuracy of the biomarker score and clinical model was determined using the area under the curve (38). The ability of the biomarker risk score to correctly reclassify patients was additionally tested with the NRI (39).

Using methods described previously, we evaluated the effect that screening patients for an elevated biomarker score would have on the total sample size of a clinical trial for an intervention intended to prevent CKD (40). Our hypothetical clinical trial included a 12-month accrual period and 36-month follow-up; it specified $90 \%$ power to detect an HR of 0.8 using 2-sided testing and $\alpha$ equal to 0.05 .

For our mouse model, multigroup comparison was performed by 1-way analysis of variance (ANOVA) for group mean comparison followed by Tukey's multiple-comparison test for subgroup comparison. Two-model time-course comparison was performed by 2-way ANOVA to test whether there was a difference between the models and in the time course, followed by Bonferroni's post hoc test for subgroup comparison at each time point. Correlation of gene expression was determined by Pearson's correlation coefficient.

Analyses were conducted using SAS software, version 9.4, Prism 8 (GraphPad Software), and R version 4.0.2. All tests of significance were 2-sided, with $P$ less than 0.05 considered significant.

Study approval. The study was approved by the institutional review boards of all participating institutions, and written informed consent was obtained from all participants. All animal protocols were approved by the Yale University Animal Care and Use Committee.

\section{Author contributions}

JP, HTP, LX, LGC, and CRP drafted the manuscript. JP, HTP, and LX analyzed the data. All authors participated in the conception and design of the study, acquisition or interpretation of the data, critical revision of the article for important intellectual content, and final approval of the version to be published.

\section{Acknowledgments}

The authors would like to thank all of the ASSESS-AKI study participants, research coordinators, and support staff for making this study possible. The ASSESS-AKI was supported by cooperative agreements from the NIDDK (U01DK082223, U01DK082185, U01DK082192, and U01DK082183). We also acknowledge funding support from NIH grants R01HL085757, R01DK098233, R01DK101507, R01DK114014, K23DK100468, R03DK111881, R01DK093771, K01DK120783, P30DK079310, and P30DK114809.

Address correspondence to: Chirag R. Parikh, Division of Nephrology, Ronald Peterson Professor of Medicine, Johns Hopkins University School of Medicine, 1830 E. Monument Street, Suite 416, Baltimore, Maryland 21287, USA. Phone: 410.955.5268; Email: chirag.parikh@jhmi.edu.
1. Chawla LS, et al. Acute kidney injury and chronic kidney disease as interconnected syndromes. NEngl JMed. 2014;371(1):58-66.

2. Ishani A, et al. Acute kidney injury increases risk of ESRD among elderly. J Am Soc Nephrol. 2009;20(1):223-228.

3. Wald R, et al. Chronic dialysis and death among survivors of acute kidney injury requiring dialysis. JAMA. 2009;302(11):1179-1185.

4. Ferenbach DA, Bonventre JV. Mechanisms of maladaptive repair after AKI leading to accelerated kidney ageing and CKD. Nat Rev Nephrol. 2015;11(5):264-276.

5. Xu K, et al. Unique transcriptional programs identify subtypes of AKI. JAm Soc Nephrol. 2017;28(6):1729-1740.

6. Hall IE, et al. Urine YKL-4O is associated with progressive acute kidney injury or death in hospi- talized patients. BMC Nephrol. 2014;15:133.

7. Huen SC, Parikh CR. Molecular phenotyping of clinical AKI with novel urinary biomarkers. Am J Physiol Renal Physiol. 2015;309(5):F406-F413.

8. Puthumana J, et al. YKL-40 associates with renal recovery in deceased donor kidney transplantation. J Am Soc Nephrol. 2017;28(2):661-670.

9. Schmidt IM, et al. Chitinase-like protein Brp-39/ YKL-40 modulates the renal response to ischemic injury and predicts delayed allograft function. J Am Soc Nephrol. 2013;24(2):309-319.

10. Munshi R, et al. MCP-1 gene activation marks acute kidney injury. JAm Soc Nephrol. 2011;22(1):165-175.

11. Furuichi K, et al. CCR2 signaling contributes to ischemia-reperfusion injury in kidney. J Am Soc Nephrol. 2003;14(10):2503-2515.

12. Garimella PS, et al. Association of preoperative urinary uromodulin with AKI after cardiac sur- gery. Clin J Am Soc Nephrol. 2017;12(1):10-18.

13. Pruijm M, et al. Associations of urinary uromodulin with clinical characteristics and markers of tubular function in the general population. Clin J Am Soc Nephrol. 2016;11(1):70-80.

14 . Montgomery TA, et al. Breast regression protein-39/chitinase 3-like 1 promotes renal fibrosis after kidney injury via activation of myofibroblasts. J Am Soc Nephrol. 2017;28(11):3218-3226.

15. Xu L, et al. Tubular GM-CSF Promotes late MCP-1/CCR2-mediated fibrosis and inflammation after ischemia/reperfusion injury. JAm Soc Nephrol. 2019;30(10):1825-1840.

16. Le Clef $\mathrm{N}$, et al. Unilateral renal ischemia-reperfusion as a robust model for acute to chronic kidney injury in mice. PLoS One. 2016;11(3):e0152153.

17. Basile DP, et al. Progression after AKI: understanding maladaptive repair processes to predict 


\section{CLINICAL MEDICINE}

and identify therapeutic treatments. JAm Soc Nephrol. 2016;27(3):687-697.

18. Urban ML, et al. Fibrosis---a common pathway to organ injury and failure. $N$ Engl J Med. 2015;373(1):95-96.

19. Meng XM, et al. Inflammatory processes in renal fibrosis. Nat Rev Nephrol. 2014;10(9):493-503.

20. Tang PM, et al. Macrophages: versatile players in renal inflammation and fibrosis. Nat Rev Nephrol. 2019;15(3):144-158.

21. Darisipudi MN, et al. Uromodulin triggers IL-1 $\beta$ dependent innate immunity via the NLRP3 inflammasome. J Am Soc Nephrol. 2012;23(11):1783-1789.

22. Bates JM, et al. Tamm-Horsfall protein knockout mice are more prone to urinary tract infection: rapid communication. Kidney Int. 2004;65(3):791-797.

23. Gudbjartsson DF, et al. Association of variants at UMOD with chronic kidney disease and kidney stones-role of age and comorbid diseases. PLOS Genet. 2010;6(7):e1001039.

24. Ahluwalia TS, et al. Uromodulin gene variant is associated with type 2 diabetic nephropathy. J Hypertens. 2011;29(9):1731-1734.

25. US Food and Drug Administration. Enrichment strategies for clinical trials to support determination of effectiveness of human drugs and biological products guidance for industry. https://www. fda.gov/media/121320/download. Accessed
December 2, 2020.

26. Brasen JH, et al. Macrophage density in early surveillance biopsies predicts future renal transplant function. Kidney Int. 2017;92(2):479-489.

27. Park WD, et al. Fibrosis with inflammation at one year predicts transplant functional decline. J Am Soc Nephrol. 2010;21(11):1987-1997.

28. Toki D, et al. The role of macrophages in the development of human renal allograft fibrosis in the first year after transplantation. Am J Transplant. 2014;14(9):2126-2136.

29. Centers for Disease Control and Prevention. Chronic kidney disease in the United States. 2019. https://www.cdc.gov/kidneydisease/ pdf/2019_National-Chronic-Kidney-DiseaseFact-Sheet.pdf. Accessed December 2, 2020.

30. Go AS, et al. The assessment, serial evaluation, and subsequent sequelae of acute kidney injury (ASSESS-AKI) study: design and methods. BMC Nephrol. 2010;11:22.

31. Ikizler TA, et al. A prospective cohort study that examined acute kidney injury and kidney outcomes, cardiovascular events and death informs on long-term clinical outcomes. Kidney Int. 2020;99(2):456-465.

32. Levey AS, et al. A new equation to estimate glomerular filtration rate. Ann Intern Med. 2009;150(9):604-612.

33. Parikh CR, et al. Kidney biomarkers of injury and repair as predictors of contrast-associated AKI: a substudy of the PRESERVE trial. Am J Kidney Dis. 2019;75(2):187-194.

34. Butler A, et al. Integrating single-cell transcriptomic data across different conditions, technologies, and species. Nat Biotechnol. 2018;36(5):411-420.

35. Kang HM, et al. Multiplexed droplet single-cell RNA-sequencing using natural genetic variation. Nat Biotechnol. 2018;36(1):89-94.

36. Hayek SS, et al. Soluble urokinase receptor and chronic kidney disease. N Engl J Med. 2015;373(20):1916-1925.

37. Ruff CT, et al. Cardiovascular biomarker score and clinical outcomes in patients with atrial fibrillation: a subanalysis of the ENGAGE AF-TIMI 48 randomized clinical trial. JAMA Cardiol. 2016;1(9):999-1006.

38. DeLong ER, et al. Comparing the areas under two or more correlated receiver operating characteristic curves: a nonparametric approach. Biometrics. 1988;44(3):837-845.

39. Pencina MJ, et al. Evaluating the added predictive ability of a new marker: from area under the ROC curve to reclassification and beyond. Stat Med. 2008;27(2):157-172; discussion 207-212.

40. Cheng S, et al. BioPETsurv: Methodology and open source software to evaluate biomarkers for prognostic enrichment of time-to-event clinical trials. PLoS One. 2020;15(9):e0239486. 